\title{
The effect of pycnogenol on lymphatic nodes and adhesion during in a peritoneal adhesion model in rats ${ }^{1}$
}

\author{
Ceren Canbey Goret', Nuri Emrah Goret", Asli Kiraz"I', Omer Faruk Ozkan"1v, Muammer Karaayvaz
}

'Assistant Professor, Department of Surgical Pathology, Health Sciences University, Sancaktepe Research and Education Hospital, Istanbul, Turkey. Scientific, intellectual, conception and design of the study; analysis and interpretation of data; histopathological examinations; technical procedures; statistical analysis; manuscript preparation and writing.

"Assistant Professor, Department of General Surgery, Canakkale State Hospital, Turkey. Conception and design of the study, acquisition of data, technical procedures, statistical analysis, statistical analysis, manuscript preparation.

I'Issistant Professor, Departments of Microbiology, Onsekiz Mart University Research and Application Hospital, Canakkale, Turkey. Scientific and intellectual content of the study, manuscript preparation.

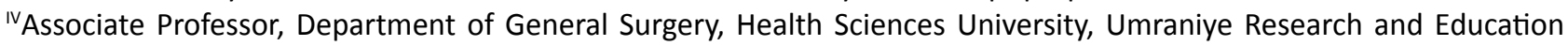
Hospital, Istanbul, Turkey. Acquisition, analysis and interpretation of data; statistical analysis; manuscript preparation and writing; critical revision; final approval.

vProfessor, Department General Surgery, Onsekiz Mart University Research and Application Hospital, Canakkale, Turkey. Critical revision, final approval.

\section{Abstract}

Purpose: To investigate the effects of pycnogenol on peritoneal adhesions and additionally to investigate the immunohistochemical effects of free oxygen radicals and reactive lymph nodes detected in the adhesive tissue that was sampled surrounding the cecum on intraabdominal adhesions.

Methods: Twenty-seven Wistar Albino rats were divided into three groups. In group 1 (sham), laparotomy was performed and stitched up. In group 2 (control), after laparotomy was performed, punctate hemorrhage was induced by cecal abrasion in the cecum and each rat was intraperitoneally administered 2 cc of saline. In group 3 (experimental), after laparotomy was performed, punctate hemorrhage was induced by cecal abrasion in the cecum and each rat was intraperitoneally administered a sterile Pycnogenol derivative. The rats in all groups were re-laparotomized on postoperative day 7 ; samples were obtained from the peritoneal tissue surrounding the cecum, and the rats were sacrificed.

Results: In group 3, there was a statistically significant difference in terms of inflammation, lymph node size, and free oxygen radicals; these parameters tended to increase. In terms of fibrosis evaluated using $\mathrm{H} \& \mathrm{E}$ and MT, there was no significant difference between groups 2 and 3.

Conclusions: No positive outcomes indicating that pycnogenol reduces intra-abdominal adhesions were obtained. However, it caused severe inflammation in the tissue. Moreover, a significant increase in lymph node size was detected secondary to inflammation. Additionally, in immunohistochemical analyses conducted to detect oxidative stress, pycnogenol increased the production of free oxygen radicals in the tissue.

Key words: Pycnogenol. Lymphadenopathy. Free Radicals. Rats. 


\section{Introduction}

Today, intra-abdominal adhesions occurring in the postoperative period have become more common due to the increasing number of abdominal operations performed as a result of development in anesthesia and surgical techniques. Peritoneal adhesions are one of the most significant problems to be overcome via surgical procedures ${ }^{1,2}$. Researchers have conducted studies for preventing complications that may result in morbidity, particularly intestinal obstruction and infertility ${ }^{3}$. These complications are serious and may require reoperation. Reoperations may cause high morbidity and mortality rates. The etiopathogenesis of peritoneal adhesions is not precisely known; however, inflammation, ischemia, and the presence of oxidative stress may be prevalent its etiopathogenesis ${ }^{2,4-6}$. Many types of steroids and non-steroidal antiinflammatory, fibrinolytic, and antioxidant agents as well as different techniques have been used to reduce or prevent the formation of postoperative peritoneal adhesions s.5,7. $^{25,}$ Additionally, conditions such as inflammation and infection that are secondary to intraabdominal operations may cause adhesions, whereas they may be responsible for the etiopathogenesis of lymphadenopathy that may develop secondary to inflammation ${ }^{2,7}$. In the present study, we aimed to investigate the effects of Pycnogenol, also known as Pinus pinaster bark extract, on peritoneal adhesions; the antioxidant, antimicrobial, and antiinflammatory effects of Pycnogenol have been reported in the literature in terms of preventing and reducing intra-abdominal adhesions that

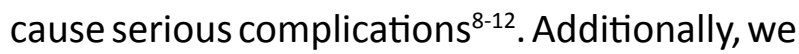
aimed to investigate the immunohistochemical effects of free oxygen radicals and reactive lymph nodes detected in the adhesive tissue that was sampled surrounding the cecum on intra-abdominal adhesions.

\section{Methods}

\section{Experimental protocol}

This study was performed in the laboratory of Canakkale Onsekiz Mart University Health Sciences Research and Application Center, Turkey, with the approval of the university's Laboratory Animals Ethics Committee (No: 2017/27348).

All rats obtained from the Laboratory Animal Research Center of Canakkale Onsekiz Mart University, were used in this study and were housed in special metal cages at room temperature under stable environmental conditions $\left(21 \pm 2^{\circ} \mathrm{C}, 12\right.$-h light/dark cycle). The rats were given access to normal water and standard food, with no restriction. Before the experimental procedure, all rats were weighed with an analytical balance and body weights (BW) were recorded. Wistar albino rats weighing 200-240 g were divided into three groups (in each group, $\mathrm{n}=9$ ). All rats were anesthetized with intramuscular (IM) ketamine (Ketalar 500 mg, 90 mg/kg body weight [BW]; Pfizer) and xylazine (Kepro Xylazine 20, $10 \mathrm{mg} /$ kg BW; Biopharm Veterinary Drugs).

Group 1 (sham group): Rats were laparotomized and stitched up under anesthesia. On postoperative day 7 , the rats were relaparotomized under anesthesia; the peritoneal tissue surrounding the cecum was sampled, and the rats were sacrificed.

Group 2 (control group: physiological saline solution): After rats were laparotomized under anesthesia, punctate hemorrhage was induced by cecal abrasion in the cecum (Figure 1); each rat was intraperitoneally administered 2 cc of saline solution. On postoperative day 7 , the rats were relaparotomized under anesthesia; the peritoneal tissue surrounding the cecum was sampled, and the rats were sacrificed.

Group 3 (experimental group: Pycnogenol): After rats were laparotomized 
under anesthesia, punctate hemorrhage was induced by cecal abrasion in the cecum; each rat was intraperitoneally administered a sterile Pycnogenol derivative diluted with $10 \mathrm{mg} /$ $\mathrm{kg}$ saline. On postoperative day 7 , the rats were relaparotomized under anesthesia; the peritoneal tissue surrounding the cecum was sampled, and the rats were sacrificed.

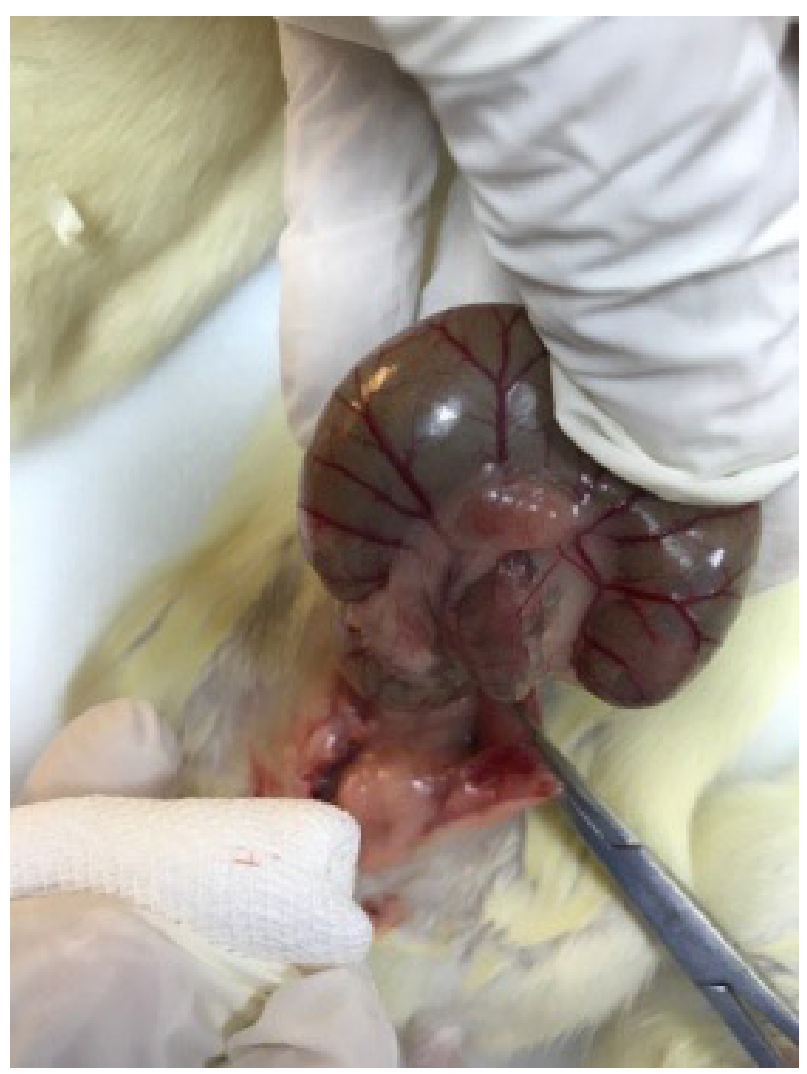

Figure 1 - Inducing punctate hemorrhage in the cecum by cecal abrasion after laparotomy.

\section{Histological examination}

All specimens were fixed with $10 \%$ buffered formaldehyde. Specimens embedded in paraffin blocks were stained with hematoxylin and eosin (H\&E) by taking 4-5- $\mu \mathrm{m}$ thick sections. In addition to Hematoksilen \& Eozin (H\&E), specimens were histochemically stained with Masson's Trichrome (MT) by taking 3-4- $\mu \mathrm{m}$-thick sections. In addition, immunohistochemical staining was performed using Glutathione S-transferase (GSTP-1), Glutathione Reductase (Glut Red), Superoxide dismutase (SOD1) and Catalase (CAT) with DAKO kits in a DAKO automatic staining device by taking $3-\mu m$-thick sections from paraffin blocks.

Histopathologically, the following parameters were evaluated (Chart 1):

- Fibrosis

- Inflammation

- Number of lymph nodes

- Size of the largest lymph node

The effects of fibrosis were evaluated as follows:

No effect: 0; Minor effect: 1; Moderate effect: 2; Intense effect: 3.

GSTP-1, SOD-1, CAT, and Glut Red staining were evaluated as follows:

0 : no staining; 1 : weak positivity; 2 : moderate positivity; 3 : strong positivity;

Histochemical fibrosis assessments performed using MT were evaluated as follows:

0 : no staining; 1 : weak positivity; 2 : moderate positivity; 3 : strong positivity.

Chart 1 - Histopathological scoring table.

\begin{tabular}{lllll}
\hline Score & Fibrosis & Inflamation & Masson Tricrom & $\begin{array}{l}\text { SOD1 } \\
\text { CAT } \\
\text { GSTP1 } \\
\end{array}$ \\
& & & $\begin{array}{l}\text { GLUT RED } \\
\text { (Immunohistochemistry) }\end{array}$ \\
& & & None \\
$\mathbf{0}$ & None & None & None & Mild \\
$\mathbf{1}$ & Mild & Mild & Mild & Significant \\
$\mathbf{2}$ & Significant & Significant & Significant & Intense \\
$\mathbf{3}$ & Intense & Intense & Intense & \\
\hline
\end{tabular}




\section{Statistical analysis}

The data were analyzed by the IBM SPSS 20 program. The normal distribution of the variables was investigated with visual (histogram and probability plots) and analytical methods taking account of sample size (Kolmogorow Smirnow test). As all variables did not have normal distribution, the KruskalWallis test was used for comparisons between groups. Descriptive statistics are given as arithmetic mean \pm standard deviation and median (quartiles). A $P<0.05$ was accepted as statistically significant. For pairwise comparisons, the One-Way ANOVA test was used. Differences among groups and changes in course of time for number of lymph nodes and total scores variants were analyzed with two way variance analysis. Bonferroni test was used for the differences among groups. Statistical significance level was accepted as $P$ $<0.05$.

\section{- Results}

From statistical analyses, the results of specimens in groups 1, 2, and 3 were not normally distributed; therefore, they were compared using the Kruskal-Wallis test.

-Inflammation (Figures 2 and 4), fibrosis, lymph node size (Figure 3) and histochemical fibrosis assessment using MT (Figure 5) parameters were evaluated by the Mann-Whitney $U$ test in pair-group analysis. The asymptomatic sign value between groups 1,2 , and 3 for each parameter was $P 0.001$ ( $P$ $<0.05)$; and there was a statistical difference between all groups.

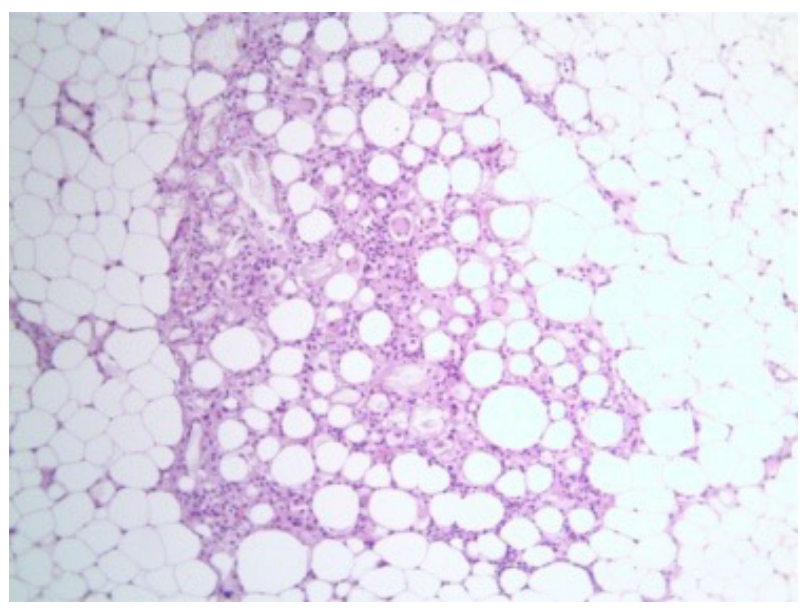

Figure 2 - Foreign body reaction against Pycnogenol accompanied by severe inflammation and osteoclastic giant cells in mature adipose tissue (hematoxylin-eosin staining $\times 100$ ).

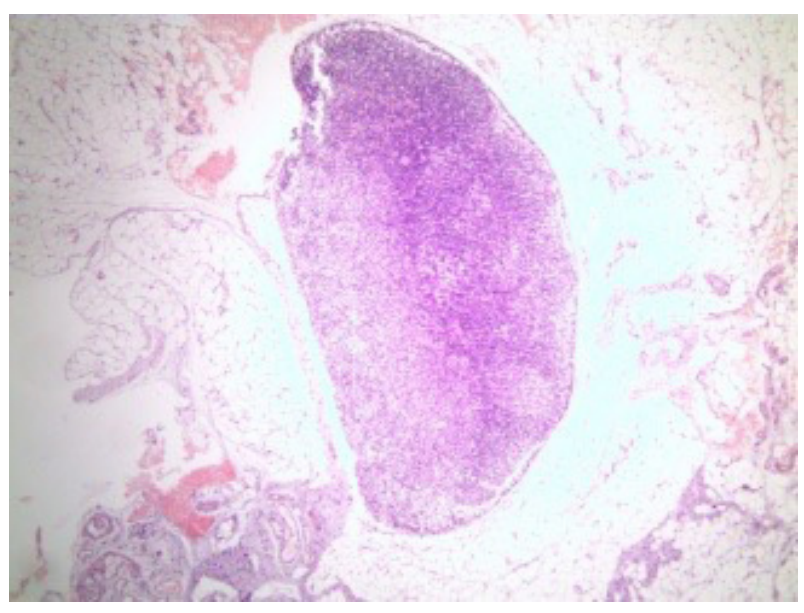

Figure 3 - Reactive lymphoid hyperplasia in mature adipose tissue (hematoxylin-eosin staining $\times 40$ ).

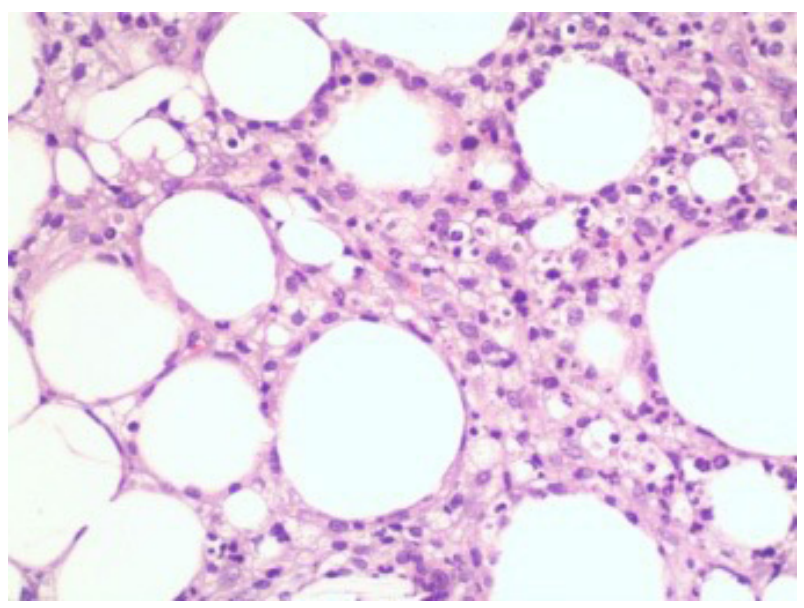

Figure 4 - Severe inflammation (score 3 ) in mature adipose tissue (hematoxylin-eosin staining $\times 400$ ). 


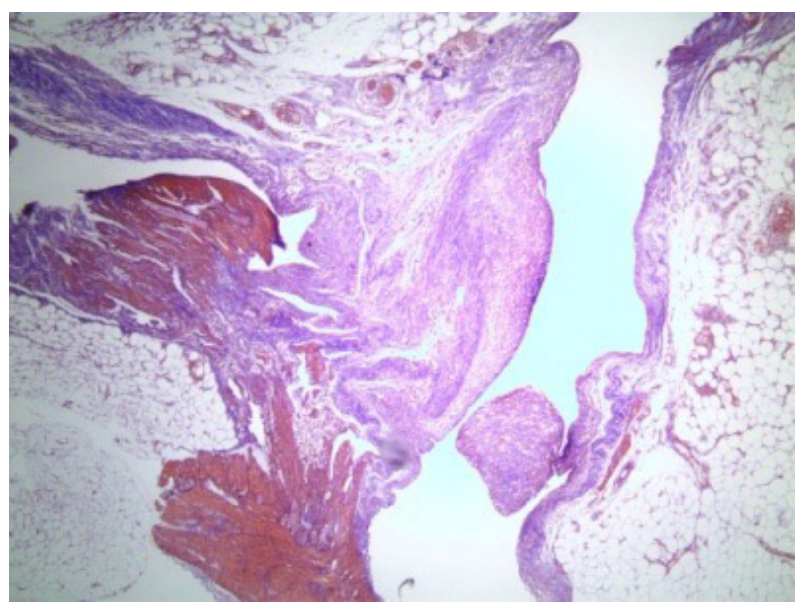

Figure 5 - Strong positivity (score 3) with Masson's trichrome (Histochemistry Masson's trichrome staining $\times 40$ ).

-For fibrosis: $(P<0.05)$

$P$-value between groups 1 and 2 was 0.001 and statistically significant.

$P$-value between groups 1 and 3 was 0.001 and statistically significant.

$P$-value between groups 2 and 3 was 0.730 and was not statistically significant.

-For inflammation: $(P<0.05)$

$P$-value between groups 1 and 2 was 0.005 and statistically significant.

$P$-value between groups 1 and 3 was 0.001 and statistically significant.

$P$-value between groups 2 and 3 was 0.002 and statistically significant.

-For histochemical assessment performed with MT: $(P<0.05)$

$P$-value between groups 1 and 2 was 0.001 and statistically significant.

$P$-value between groups 1 and 3 was 0.001 and statistically significant.

$P$-value between groups 2 and 3 was 0.996 and was not statistically significant.

- In terms of lymph node size between the groups: $(P<0.05)$

$P$-value between groups 1 and 2 was 0.003 and statistically significant.

$P$-value between groups 1 and 3 was 0.001 and statistically significant.

$P$-value between groups 2 and 3 was 0.024 and statistically significant.
-The total immunohistochemical score and number of lymph nodes were normally distributed, and statistical evaluation between the three groups was done using oneway ANOVA.

-- In terms of the total immunohistochemical score:

The mean values were 2.33 in group 1 , 7.44 in group 2, and 7.89 in group 3.

The $P$-value was $0.0001(P<0.005)$, and there was a significant difference.

In immunohistochemical analyses (SOD-1, Glut Red, CAT, GSTP-1) on free oxygen radicals, Pycnogenol statistically increased the production of free oxygen radicals in tissues (Figures 6 to 10).

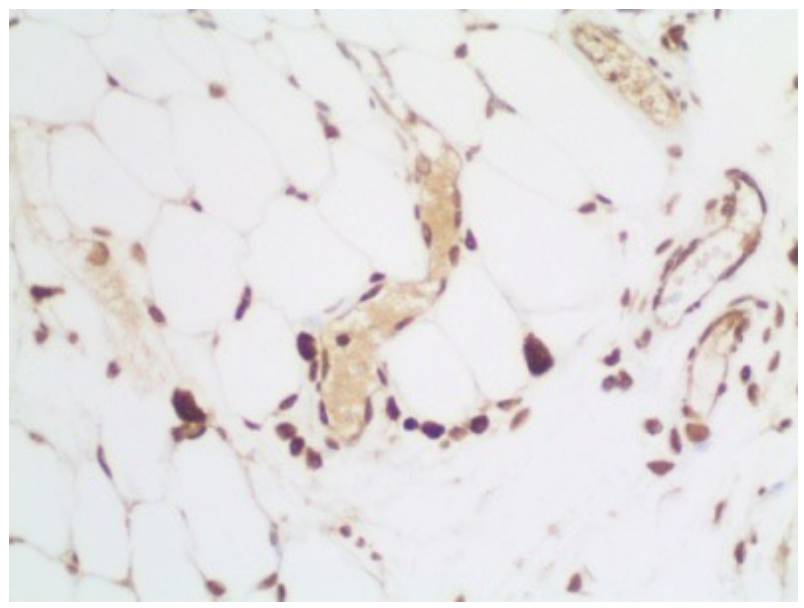

Figure 6 - Strong staining (score 3) with SOD-1 in adipose tissue (immunohistochemistry SOD-1 staining $\times 400$ ).

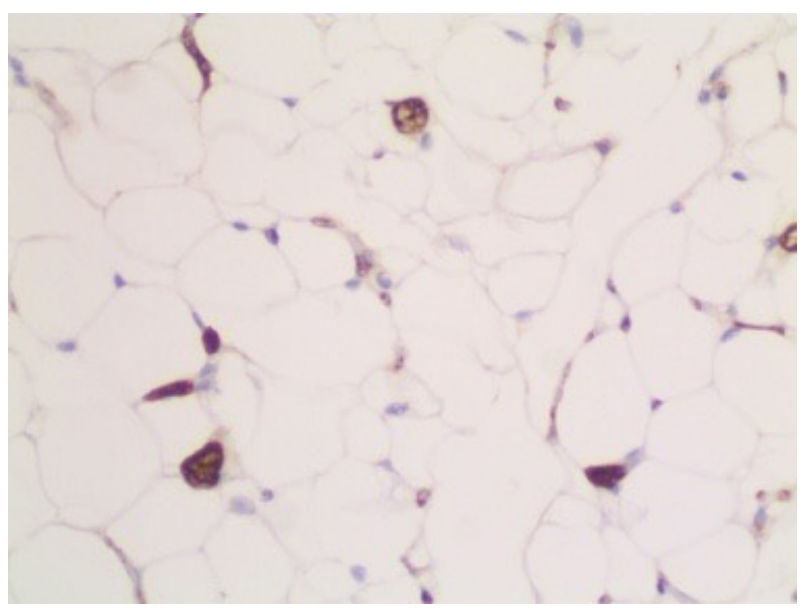

Figure 7 - Moderate positivity (score 2) with GSTP-1 (immunohistochemistry GSTP-1 staining $\times 400$ ). 


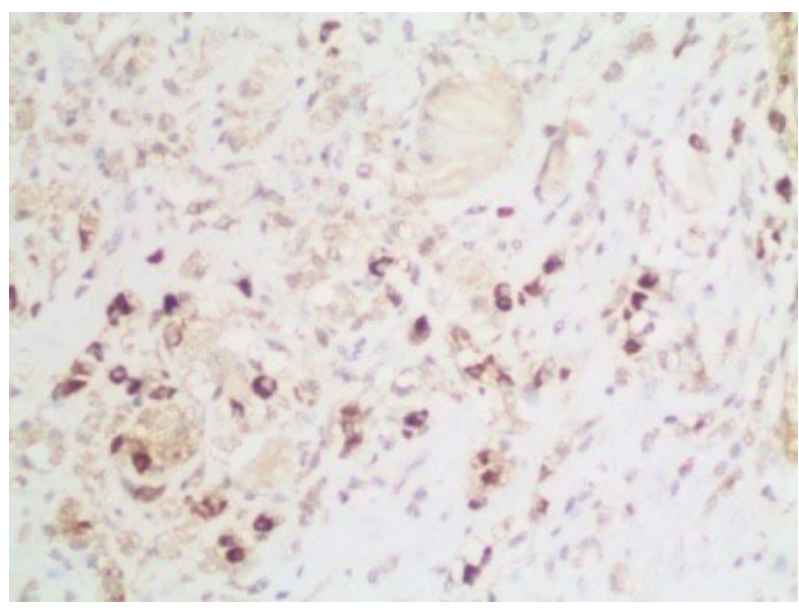

Figure 8 - Strong staining (score 3) with CAT (immunohistochemistry CAT staining $\times 400$ ).

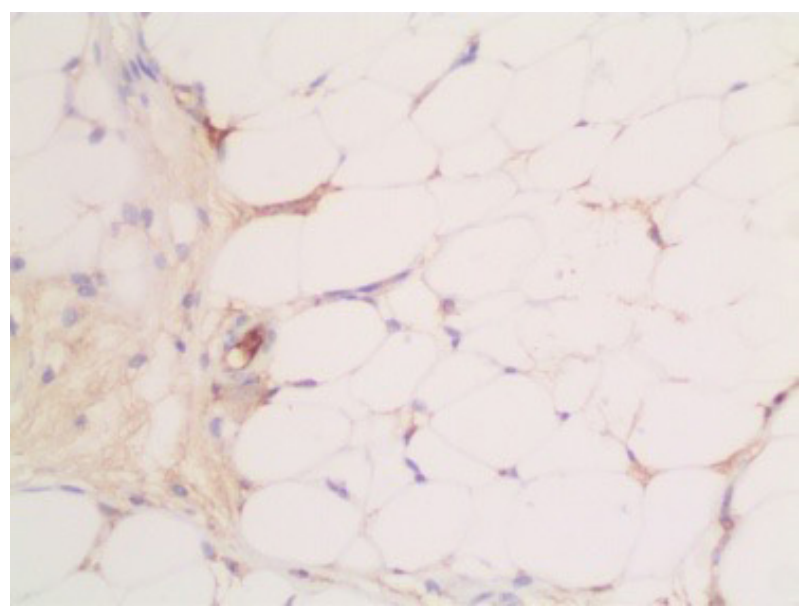

Figure 9 - Weak staining (score 1) with CAT (immunohistochemistry CAT staining $\times 400$ ).

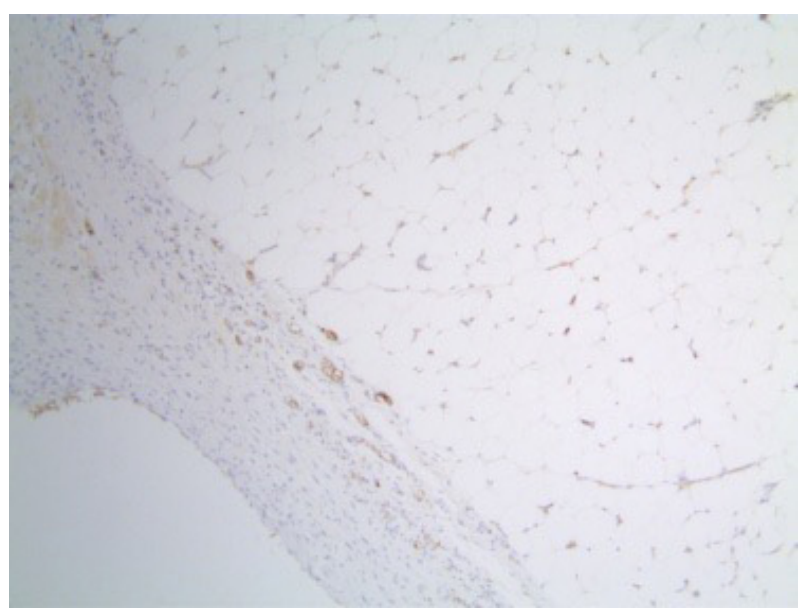

Figure 10 - Moderate staining (score 2) with Glut Red (immunohistochemistry Glut Red staining $\times 40$ ).

- In terms of number of lymph nodes:

The means values were 3.1111 in group $1,3.6667$ in group 2, and 1.4444 in group 3.

The $P$-value was $0.017(P<0.005)$, and there was no statistically significant difference.

In terms of the total immunohistochemical score and number of lymph nodes, post hoc pair-group analyses were evaluated using Bonferroni correction $* * *$. (The mean difference was significant at the level of 0.15 .) $(* * *$ In terms of the total immunohistochemical score, the $P$-value was 0.001 between groups 1 and 2, 0.0001 between groups 1 and 3, and 0.996 between groups 2 and $3 . * * *$ In terms of the number of lymph nodes, the $P$-value was 0.996 between groups 1 and 2, 0.101 between groups 1 and 3, and 0.018 between groups 2 and 3.). Tables 1 to 3 list all evaluated parameters.

Table 1 - Histopathological evaluation of the study groups.

\begin{tabular}{|c|c|c|c|c|}
\hline Groups & Fibrosis & $\begin{array}{l}\text { Fibrosis with Masson's } \\
\text { trichrome }\end{array}$ & Inflammation & Lymph node size \\
\hline Group 1-2 & $P 0.001$ & $P 0.001$ & $P 0.005$ & $P 0.003$ \\
\hline Group 1-3 & $P 0.001$ & $P 0.001$ & $P 0.001$ & $P 0.001$ \\
\hline Group 2-3 & $P 0,730$ & $P 0,996$ & $P 0,002$ & $P 0,024$ \\
\hline
\end{tabular}

${ }^{*} P<0.05$, pair-group analysis was evaluated via the Mann-Whitney $U$ test. **Group 1: sham group, group 2: control group, group 3: experimental group. $* *$ Lymph node size was microscopically measured in the histopathological examination in $\mathrm{mm}$. 
Table 2 - Comparison between the groups with respect to the immunohistochemical score and the number of lymph nodes.

\begin{tabular}{lll}
\hline Groups & Number of lymph nodes & Immunohistochemical score \\
\hline Group 1-2 & 0,996 & 0.001 \\
Group 1-3 & 0.101 & 0.0001 \\
Group 2-3 & 0.018 & 0,996 \\
\hline
\end{tabular}

*The mean difference is significant at the level of 0.15 . ** Post hoc pair-group analysis was performed using Bonferroni correction. $* * *$ Statistical evaluation between the three groups was assessed with one-way ANOVA. **** Each immunohistochemical marker was individually scored, but statistical evaluation was performed for each rat on the basis of the total score (SOD-1, CAT, GSTP-1, Glut Red).

$* * * * *$ In the histopathological examination, the number of lymph nodes was microscopically counted for each rat.

Table 3 - Mean values of free oxygen radicals and number of lymph nodes.

\begin{tabular}{lll}
\hline Groups & Number of lymph nodes & Free oxygen radicals with immunohistochemical \\
\hline Group 1 & Mean: 3,1111 & Mean: 2,33 \\
& St. deviation: 1,16667 & St. deviation: 1,5 \\
Group 2 & Median: 3 & Median: 2 \\
& St. deviation: 1,58114 & Mean: 7,44 \\
& Median: 3 & St. deviation: 1,59 \\
Group 3 & Mean: 1,4444 & Median: 8 \\
& St. deviation: 1,87824 & Mean: 7,89 \\
& Median: 1 & St. deviation: 1,833 \\
\hline
\end{tabular}

*The mean difference is significant at the level of 0.15 . ** Post hoc pair-group analysis was performed using Bonferroni correction. *** Statistical evaluation between the three groups was assessed with one-way ANOVA. **** Each immunohistochemical marker was individually scored, but statistical evaluation was performed for each rat on the basis of the total score (SOD-1, CAT, GSTP-1, Glut Red). $* * * * *$ In the histopathological examination, the number of lymph nodes was microscopically counted for each rat.

\section{Discussion}

At present, peritoneal adhesions are still one of the most important complications to be overcome in surgical procedures. Bleeding, cauterization, ischemia, infections, and conditions that may damage the peritoneum may trigger adhesions ${ }^{5-7}$. While numerous cells such as mesothelial cells, histiocytes, fibroblasts, and lymphocytes help in healing the injured area, they also play a role in the development of peritoneal adhesion ${ }^{2-5}$. It has been demonstrated that adhesions are often the result of improper healing due to injured peritoneal tissues associated with oxidative stress $^{2}$. In our study, in addition to parameters such as fibrosis and inflammation, we performed GSTP-1, Glut Red, SOD-1, and CAT staining to immunohistochemically examine oxidative stress parameters in tissues. After postoperative days 5 and 7 , these adhesive strips progressed to persistent fibrous adhesions ${ }^{2,13}$. It was also reported that hypoxia directly promotes the production of free oxygen radicals in tissues ${ }^{2,14}$. With the increase in the production of free oxygen radicals, the production of fibroblasts, tissue growth factor, vascular endothelial growth factor, and cytokines such as cyclooxygenase- 2 that cause adhesions also increased ${ }^{14,15}$. As a result, histopathologically, inflammation and fibrosis occur $^{16-18}$. There are studies indicating that fibroblasts reduce antioxidant levels similar to free oxygen radicals ${ }^{14-18}$. If the production of free oxygen radicals was reduced using antioxidants, adhesive fibroblasts transformed 
into normal fibroblasts and collagen production decreased $^{15}$. There are several antioxidant agents, and many studies were conducted using them. Keleidari et al. ${ }^{19}$ evaluated the effect of vitamin $C$ on postoperative peritoneal adhesions and reported better wound healing and decreased adhesion formation in the experimental group than in the control group. The efficacy of vitamin $E$ in the prevention of post-operative peritoneal adhesion has been reported $^{1,20,21}$. Şahbaz et al. ${ }^{2}$ demonstrated that pycnogenol, which is an antioxidant, was effective for preventing peritoneal adhesion on postoperative day 10 owing to its anti-inflammatory and antioxidant effects. However, in contrast to the results of this study, we found that pycnogenol severely increased inflammation and resulted in foreign body reaction in some rats. In the present study, when we consider fibrosis using $H$ \& $E$ and $\mathrm{MT}$, there was a statistically significant increase in groups 2 and 3 compared to group 1. However, no significant difference was found between groups 2 and 3 . Oxidative stressinduced cellular damage triggers adhesion. In our study, in immunohistochemical analyses performed on tissues taken on day 7 to detect oxidative stress using GSTP-1, Glut Red, SOD1 , and CAT, it was found that Pycnogenol statistically increased free oxygen radicals in the tissues. In a study conducted by Noda et $a .^{22}$, pycnogenol was reported to be one of the effective free radical scavengers. However, in our study, immunohistochemical analyses revealed that the production of free oxygen radicals increased in the tissue. We believe that the reason for this was that the intraperitoneal administration of pycnogenol was effective for increasing inflammation. In a study conducted on the efficacy of pycnogenol in wound healing in diabetic rats, it was reported that Pycnogenol is a feasible option to accelerate wound healing ${ }^{23}$. In a study using an experimental sepsis model in rats, it was reported that the production of free oxygen radicals in rats treated with Pycnogenol was significantly reduced $^{24}$. In a study conducted in rats, it was reported that Pycnogenol facilitated the healing of colon anastomoses ${ }^{25}$. In the present study, in addition to these histopathological findings, many reactive lymphadenopathies were detected in various sizes in almost all groups. Reactive lymphadenopathies usually occur secondary to infections, inflammation, or malignancy. Lymph nodes have functions such as microorganism filtration and antibody production. Lymph nodes grow as in the presence of microorganisms, malignant cells, or antigenic reaction, they result in the proliferation of lymphocytes or macrophage hyperplasia ${ }^{26}$. In acute inflammation, lymph nodes grow due to cellular infiltration and edema. Similarly, in chronic inflammation, lymph nodes also grow; however, mononuclear cell infiltration is present ${ }^{26}$. In our study, we believe that the presence of acute inflammation, which later becomes chronic, plays a role in the etiology of lymphoid hyperplasia, which was statistically significant, particularly in group 3. The foreign body reaction against Pycnogenol sporadically triggers inflammation and lymphoid hyperplasia. This is one of the positive results we obtained, and we plan to conduct studies to reveal the causes of this result. In the present study, we did not find a significant difference between the groups in terms of lymph node numbers.

\section{- Conclusions}

Pycnogenol did not result in a reduction in intra-abdominal adhesions; rather, it led to severe inflammation in the tissue. Moreover, we found a significant increase in lymph node size secondary to inflammation. Additionally, in the immunohistochemical analyses, we found that Pycnogenol statistically increased the production of free oxygen radicals in the 
tissue. Although Pycnogenol was reported to have a positive effect on peritoneal adhesions in the literature, in the present study, we found inflammation in the early phase and a slight increase in the production of free oxygen radicals and the size of lymph nodes. We believe that this effect is due to the fact that we terminated the experimental model early (day 7). Further studies are needed to evaluate the effects of Pycnogenol in the prevention of adhesion.

\section{References}

1 Durmus AS, Yildiz H, Yaman I, Simsek H. Efficacy of vitamin $E$ and selenium for the prevention of intraabdominal adhesions in rats: uterine horn models. Clinics. 2011;66:1247-251. doi: 10.1590/S180759322011000700021.

2 Sahbaz A, Aynioglu O, Isik H, Gun BD, Cengil O, Erol O. Pycnogenol prevents peritoneal adhesions. Arch Gynecol Obstet. 2015;292:1279. doi: 10.1007/s00404-0153764-4.

3 Brochhausen C, Schmitt VH, Planck CN, Rajab TK, Hollemann D, Tapprich C, Krämer $B$, Wallwiener $C$, Hierlemann $H$, Zehbe $R$, Planck $\mathrm{H}$, Kirkpatrick CJ. Current strategies and future perspectives for intraperitoneal adhesion prevention. J Gastrointest Surg. 2012;16:1256-74. doi: 10.1007/s11605011-1819-9.

4 Fletcher NM, AbuanzehS, Saed MG, Diamond MP, AbuSoud HM, Saed GM. Nicotinamide adenine dinucleotide phosphate oxidase expression is differentially regulated to favor a prooxidant state that contributes to postoperative adhesion development. Reprod Sci. 2014;21:1050-9. doi: $10.1177 / 1933719114522524$.

5 Arung W, Meurisse M, Detry $O$. Pathophysiology and prevention of postoperative peritoneal adhesions. World J Gastroenterol. 2011;17:4545-53. doi: 10.3748/wjg.v17.i41.4545.

6 Liakakos T, Thomakos N, Fine PM, Dervenis C, Young RL. Peritoneal adhesions: etiology, pathophysiology, and clinical significance. Recent advances in prevention and management. Dig Surg. 2001;18:260-73. doi: 10.1159/000050149.

7 Kamel Remah M. Prevention of postoperative peritoneal adhesions. Eur J Obstet Gynecol Reprod Biol. 2010;150:1118. doi: 10.1016/j.ejogrb.2010.02.003.

8 Peng YJ, Lee CH, Wang CC, Salter DM, Lee HS. Pycnogenol attenuates the inflammatory and nitrosative stress on joint inflammation induced by urate crystals. Free Radic Biol Med. 2012;52:765-74. doi: 10.1016/j. freeradbiomed.2011.12.003.

9 Rohdewald PA. Review of the French maritime pine bark extract (Pycnogenol), a herbal medication with a diverse clinical pharmacology. Int J Clin Pharmacol Ther. 2002;40:158-68. PMID: 11996210.

10 Petrassi C, Mastromarino A, Spartera C. PYCNOGENOL in chronic venous insufficiency. Phytomedicine. 2000;7:383-8. doi: 10.1016/S0944-7113(00)80059-8.

11 Torras MA, Faura CA, Schönlau F, Rohdewald P. Antimicrobial activity of pycnogenol. Phytother Res. 2005;19:647-48. doi: 10.1002/ptr.1662.

12 Iravani S, Zolfaghari B. Pharmaceutical and nutraceutical effects of Pinus pinaster bark extract. Res Pharm Sci. 2011;6:1-11. PMID: 22049273.

13 Holmdahl L, Ivarsson ML. Therole ofcytokines, coagulation, and fibrinolysis in peritoneal tissue repair. Eur J Surg. 1999;165:1012-9. doi: 10.1080/110241599750007810

14 Awonuga AO, Belotte J, Abuanzeh S, Fletcher NM, Diamond MP, Saed GM. Advances in the pathogenesis of adhesion development: the role of oxidative stress. Reprod Sci. 2014;21:823-36. doi: 10.1177/1933719114522550.

15 Fletcher NM, Jiang ZL, Diamond MP, AbuSoud HM, Saed GM. Hypoxia-generated superoxide induces the development of the adhesion phenotype. Free Radic Biol Med. 2008;45:530-6. doi: 10.1016/j. freeradbiomed.2008.05.002.

16 Epstein JC, Wilson MS, Wilkosz S, Ireland G, O'Dwyer ST, Herrick SE. Human peritoneal adhesions show evidence of tissue remodeling and markers of angiogenesis. Dis Colon Rectum. 2006;49:1885-92. doi: 10.1007/s10350-006-0747-3.

17 Herrick SE, Mutsaers SE, Ozua P, Sulaiman $\mathrm{H}$, Omer A, Boulos P, Foster ML, Laurent 
GJ. Human peritoneal adhesions are highly cellular, innervated, and vascularized. J Pathol. 2000;192:67-72. doi:10.1002/1096$9896(2000) 9999: 9999<::$ A I D PATH678>3.0.CO;2-E.

18 Binnebösel $M$, Klink CD, Serno J, Jansen PL, von Trotha KT, Neumann UP, Junge K. Chronological evaluation of inflammatory mediators during peritoneal adhesion formation using a rat model. Langenbecks Arch Surg. 2011;396:371-8. doi: 10.1007/ s00423-011-0740-8.

19 Keleidari B, Mahmoudieh M, Bahrami F, Mortazavi P, Aslani RS, Toliyat SA. The effect of vitamin $A$ and vitamin C on postoperative adhesion formation: a rat model study. J Res Med Sci. 2014;19:28-32. PMID: 24672562.

20 de la Portilla F, Ynfante I, Bejarano D, Conde J, Fernández A, Ortega JM, Carranza G. Prevention of peritoneal adhesions by intraperitoneal administration of vitamin $E:$ an experimental study in rats. Dis Colon Rectum. 2004;47:2157-61. doi: 10.1007/ s10350-004-0741-6.

21 Yetkin G, Uludag M, Citgez B, Karakoc S, Polat N, Kabukcuoglu F. Prevention of peritoneal adhesions by intraperitoneal administration of vitamin $E$ and human amniotic membrane. Int J Surg. 2009;7:561-
5. doi: 10.1016/j.ijsu.2009.09.007.

22 Noda Y, Anzai K, Mori A, Kohno M, Shinmei M, Packer L. Hydroxyl and superoxide anion radical scavenging activities of natural source antioxidants using the computerized JES-FR30 ESR spectrometer system. Biochem Mol Biol Int. 1997;42:35-44. PMID: 9192083.

23 Dogan E, Yanmaz L, Gedikli S, Ersoz U, Okumus $Z$. The effect of pycnogenol on wound healing in diabetic rats. Ostomy Wound Manage. 2017;63:41-7. PMID: 28448268.

24 Taner G, Aydın S, Bacanlı M, Sarıgöl Z, Sahin T, Başaran AA, Başaran N. Modulating effects of pycnogenol on oxidative stress and DNA damage induced by sepsis in rats. Phytother Res. 2014;28:1692-1700. doi: 10.1002/ ptr.5184.

25 Değer KC, Şeker A, Özer I, Bostancı EB, Dalgıç T, Akmansu M, Ekinci Ö, Erçin $U$, Bilgihan A, Akoğlu M. The effects of Pycnogenol $^{\circ}$ on colon anastomotic healing in rats given preoperative irradiation. Int J Surg. 2013;11:983-88. doi: 10.1016/j. ijsu.2013.06.004.

26 Ferrer R. Lymphadenopathy: differential diagnosis and evaluation. Am Fam Physician. 1998;58:1313-20. PMID: 9803196.

\section{Correspondence:}

Dr. Ceren Canbey Goret

Health Sciences University, Sancaktepe

Research and Education Hospital

Department of Surgical Pathology

Floor 0, Istanbul Turkey

Phone: +90.507.950.02.12

drcerencanbey@hotmail.com

Received: Oct 16, 2017

Review: Dec 18, 2017

Accepted: Jan 19, 2018
Conflict of interest: none

Financial source: none
${ }^{1}$ Research performed at Laboratory of Canakkale Onsekiz Mart University Health Sciences Research and Application Center, Turkey. 